\title{
Effects of Pregnancy and Breastfeeding on Clinical Outcomes and MRI Measurements of Women with Multiple Sclerosis: An Exploratory Real-World Cohort Study
}

\author{
Lorena Lorefice (1) - Marzia Fronza - Giuseppe Fenu - Jessica Frau • \\ Giancarlo Coghe - Maurizio Nicola D'Alterio - Maria Antonietta Barracciu • \\ Federica Murgia $\cdot$ Stefano Angioni $\cdot$ Eleonora Cocco
}

Received: September 12, 2021 / Accepted: October 20, 2021 / Published online: October 29, 2021

(C) The Author(s) 2021

\section{ABSTRACT}

Introduction: Pregnancy represents an important event for women with multiple sclerosis (MS) and is often accompanied by post-partum disease reactivation. To date, the influence of this reproductive phase on long-term MS outcomes is still largely unexplored. The objective of the study was characterise a large real-world

L. Lorefice $(\bowtie) \cdot$ M. Fronza $\cdot$ G. Fenu $\cdot$ J. Frau •

G. Coghe - E. Cocco

Department of Medical Sciences and Public Health, Multiple Sclerosis Center, Binaghi Hospital, ATS

Sardegna, University of Cagliari, Via Is Guadazzonis

2, 09126 Cagliari, Italy

e-mail: lorena.lorefice@hotmail.it

\section{Fronza}

e-mail: marzia.fronza@gmail.com

G. Fenu

e-mail: giusefenu@gmail.com

J. Frau

e-mail: jessicafrauneuro@gmail.com

G. Coghe

e-mail: gcco@tiscali.it

E. Cocco

e-mail: ecocco@unica.it

M. N. D'Alterio · S. Angioni

Division of Obstetrics and Gynecology, Department of Surgical Sciences, University of Cagliari, Cagliari, Italy cohort of women with MS to evaluate the effects of pregnancy and breastfeeding on shortand long-term clinical and magnetic resonance imaging (MRI) outcomes while exploring the relationships with MRI measurements of brain atrophy.

Methods: MS patients with and without pregnancy were recruited. Clinical relapses and MRI activity of the year before conception versus the year after delivery were evaluated. Regression

\section{N. D'Alterio}

e-mail: mauridalte84@gmail.com

S. Angioni

e-mail: sangioni@yahoo.it

\section{A. Barracciu}

Radiology Unit, Binaghi Hospital, ATS Sardegna, Cagliari, Italy

e-mail: mariaantonietta.barracciu@atssardegna.it

\section{F. Murgia}

Clinical Metabolomics Unit, Department of Biomedical Sciences, University of Cagliari, Cagliari, Italy

e-mail: federica.murgia@unica.it 
models were performed to investigate the relationships between long-term MS outcomes (EDSS score and MRI brain measurements obtained by SIENAX software) and pregnancy and breastfeeding duration.

Results: Two hundred ten women with MS were enrolled; of them, 129 (61.4\%) had at least one pregnancy. Of all pregnancies $(n=212)$, those that occurred after MS onset (90 [42.4\%]) were examined to evaluate the short-term outcomes. A higher annualised relapse rate in the post-partum year versus the pre-conception year $(0.54 \pm 0.84$ vs. $0.45 \pm 0.71 ; p=0.04)$ was observed. A regression analysis showed that clinical activity after delivery was associated with clinical activity of the year before conception $(p=0.001)$ as well as duration of breastfeeding $(p=0.022)$. Similarly, post-partum MRI activity was associated with pre-conception MRI activity $(p=0.026)$ and shorter breastfeeding duration $(p=0.013)$. Regarding long-term outcomes, having had at least one pregnancy during MS was associated with a lower EDSS score $(p=0.021)$, while no relationships were reported with MRI measurements. Conversely, a breastfeeding duration $>$ 6 months was associated with lower white matter volume $(p=0.008)$.

Conclusions: Our study underlines the importance of considering the effects of pregnancy and breastfeeding on short- and long-term MS outcomes. In the current therapeutic landscape, pregnancy planning and treatment optimisation in the post-partum period, in particular for women who choose to breastfeed, are fundamental for the management of these biological phases so central in a woman's life.

Keywords: Multiple sclerosis; Pregnancy; Postpartum; Breastfeeding; Long-term MS outcomes; Treatment optimisation

\section{Key Summary Points}

Few studies have explored disease outcomes in women with multiple sclerosis (MS) who have become pregnant, and all studies have evaluated short-term outcomes.
This study aims to evaluate the long-term clinical and magnetic resonance imaging (MRI) outcomes of women with MS who have had pregnancies, also exploring the possible effects of breastfeeding on the disease course.

Having had pregnancies during MS was related to less disability, while lower white matter volumes were associated with breastfeeding duration.

Pregnancy planning and treatment optimisation in the post-partum period are fundamental for the management of women with MS.

\section{INTRODUCTION}

Pregnancy represents an important event in many women's lives. The profound hormonal changes that accompany this phase often impact the course of multiple sclerosis (MS). Many studies to date have focused on this topic. Studies agree that the physiological immunotolerance induced by pregnancy [1], especially during the third trimester, has a protective effect against MS relapses, with the annual relapse rate (ARR) dropping by 70\% [2-6]. These findings often refer to historical cohorts of patients not taking disease-modifying therapies (DMTs) but have been confirmed by a recent metanalysis of pregnancies of women treated with currently available DMTs [7]. Special attention should be given to those patients treated with highly effective DMTs such as natalizumab and fingolimod because pregnancy may not sufficiently protect against MS relapse after their discontinuation [8-11]. Compared with the pregnancy period, the post-partum year shows a rebound of disease activity, especially during the first 3 months after delivery, with an increase in relapse risk $[6,7]$. Several risk factors have been linked to post-partum relapses: higher pre-pregnancy disease activity, higher Expanded Disability Status Scale (EDSS) score [12], and an increased relapse rate during pregnancy [13]. Conversely, a recent study did 
not report a post-partum increase in MS activity. According to that study [14], the good disease control offered by currently available DMTs and meticulous prenatal counselling could allow most women with MS to experience pregnancy without post-partum relapses.

The role of breastfeeding in MS activity has been studied, but consensus is lacking about its impact on MS course. Most studies to date have reported a protective effect of breastfeeding against relapse $[15,16]$, while others reported no association $[17,18]$; none found a negative effect of breastfeeding on MS course [19]. Portaccio et al. recently published a useful study to help clinicians manage MS in post-partum women using patient characteristics as predictors of disease activity reactivation [20].

Notoriously, most studies published on pregnancy and MS have focused on the pregnancy and post-partum periods, assessing only short-term outcome measures; conversely, the effect of pregnancy as well as the role of breastfeeding on long-term clinical and neuroradiological disease outcomes remains largely unexplored.

Based on these considerations, the present study aimed to characterise a large real-world cohort of women with MS to evaluate the effects of pregnancy and breastfeeding on shortand long-term clinical and magnetic resonance imaging (MRI) outcomes while also exploring the relationships with MRI measurements of brain atrophy.

\section{METHODS}

This retrospective observational study included women with MS diagnosed according to 2017 revised McDonald criteria [21]. For each patient, demographics (age) and clinical data (disease course, disease duration, age at MS onset, and disability level assessed using the EDSS) were collected [12]. In addition, obstetric history (number and date of pregnancies), breastfeeding duration in months, and type of breastfeeding in the first 2 months (exclusive and non-exclusive) were recorded. Thus, clinical relapses and MRI activity, defined as new T2 lesions or gadolinium-enhanced lesions during in the year after childbirth as well as in the year before conception, and DMT exposure were recorded. In addition, for each patient, quantitative MRI evaluations were performed and whole-brain (WB), white matter (WM), and grey matter (GM) volumes were estimated at the time of the last neurological assessment. Informed consent was obtained from all participants after local ethics committee approval. The local ethics committee of ATS Sardegna, Sassari, approved the study (protocol 206/2020/ EC-18 February 2020).

\section{MRI Acquisition}

MRI images previously acquired using the same protocol at the same site by a 1.5-T Siemens Magnetom Avanto scanner (Siemens Medical Solutions, Erlangen, Germany) were processed. Brain tissue volume was measured using T1weighted (T1W) gradient echo images (MPRAGE) normalised for individual head size using SIENAX, an accurate, robust, and automated method of cross-sectional brain volume analysis [22]. WB, WM, and GM volumes were then calculated.

\section{Statistical Analysis}

All statistical analyses were performed using SPSS for Mac version 20.0 (SPSS Inc., Chicago, IL, USA). First, descriptive analysis was performed summarising the demographic, clinical, and MRI data as means (quantitative variables) or percentages (qualitative variables). The $t$-test was used to compare demographics (age), clinical data (age at MS onset, MS duration, EDSS), and MRI data (WB, WM, and GM volumes) of MS patients with and without pregnancy and categorise the pregnancies in relation to MS onset (before versus after).

To evaluate the effect of pregnancy on shortterm clinical outcomes, paired samples $t$-tests were used to assess differences in number of relapses pre- versus post-pregnancy defined as the year before conception and the year after childbirth as well as differences in EDSS score. Thus, linear regression models were performed to explore the determinants of clinical and MRI 
Table 1 Demographic and clinical features of MS patients included in the study

\begin{tabular}{ll}
\hline & MS patients (210) \\
\hline Age (mean \pm SD) years & $45.5 \pm 10.5$ \\
Age at MS onset & $30.7 \pm 9.8$ \\
MS duration (mean \pm SD) & $14.8 \pm 8.2$ \\
Relapsing course & $194(92.4 \%)$ \\
EDSS score & $2.2 \pm 1.5$ \\
Women with pregnancies & $129(61.4 \%)$ \\
Pre-MS onset & $60(46.5 \%)$ \\
During MS & $54(41.9 \%)$ \\
Pre and during MS & $15(11.6 \%)$ \\
Women with no pregnancies & $81(38.6 \%)$ \\
\hline
\end{tabular}

activity in the year after childbirth and control for MS features before pregnancy, DMT exposure, and the possible relationships with breastfeeding type (exclusive and non-exclusive) and duration. In addition, a binary regression analysis was performed to evaluate whether the choice of breastfeeding was conditioned by MS activity (ARR and MRI activity of the year before conception) or by exposure to second-line DMTs.

Finally, multiple regression analyses were performed to investigate the relationships between long-term MS outcomes (EDSS score and MRI measurements of WB, WM, and GM volumes) with pregnancy and breastfeeding duration after controlling for other demographics (age) and clinical variables (disease duration, clinical course).

For all assays, statistical significance $(p)$ was set at $<0.05$.

\section{Data Availability}

Anonymised data will be shared on request from any qualified investigator.

\section{RESULTS}

The study included 210 women with MS, 194 $(92.4 \%)$ of whom presented a relapsing course. Mean age and disease duration were $45.5 \pm 10.5$ and $14.8 \pm 8.2$ years, respectively, while mean EDSS score was $2.2 \pm 1.5$. As shown in Table 1, $129(61.4 \%)$ women reported previous term pregnancies, $60(46.5 \%)$ of which were prior to MS onset, 54 (41.9\%) of which were during MS course, and $15(11.6 \%)$ of which were both before and after MS onset. Using an independent samples $t$-test, significant differences in current age $(48.4 \pm 10.3$ vs. $40.9 \pm 8.9$ years $)$ and age at MS onset $(32.3 \pm 9.9$ vs. $27.9 \pm 9.1$ years) were reported between women with and without pregnancy $(p<0.005)$, respectively, with mean age $(54.1 \pm 9.4$ vs. $43.4 \pm 8.4)$ and age at MS onset $(39.1 \pm 9.2$ vs. $26.4 \pm 5.9)$

Table 2 Demographic and clinical differences between MS patients with and without previous pregnancy

\begin{tabular}{llcc}
\hline & $\begin{array}{l}\text { Pregnancies } \\
\text { Pre MS onset }(\mathbf{6 0})\end{array}$ & $\begin{array}{l}\text { Pregnancies } \\
\text { During MS (69) }\end{array}$ & No pregnancy $(\mathbf{8 1})$ \\
\hline Age (mean \pm SD) years & $54.1 \pm 9.4^{* *}$ & $43.4 \pm 8.4^{*}$ & $40.9 \pm 8.9$ \\
Age at MS onset & $39.1 \pm 9.2^{* *}$ & $26.4 \pm 5.9$ & $27.9 \pm 9.1$ \\
MS duration (mean \pm SD) & $15.0 \pm 7.9$ & $16.9 \pm 9.8^{*}$ & $12.9 \pm 6.3$ \\
EDSS score & $2.7 \pm 2.1^{*}$ & $1.9 \pm 1.3^{*}$ & $2.2 \pm 1.6$ \\
Follow-up duration & $12.6 \pm 1.1$ & $10.8 \pm 1.3$ & $9.2 \pm 1.6$ \\
\hline
\end{tabular}

${ }^{*}<0.05 ;{ }^{* *} p<0.005$

Independent $t$-test was used to compare demographic and clinical data of MS patients with previous pregnancies (categorised as pre-MS onset and during MS) vs. MS patients without previous pregnancy 


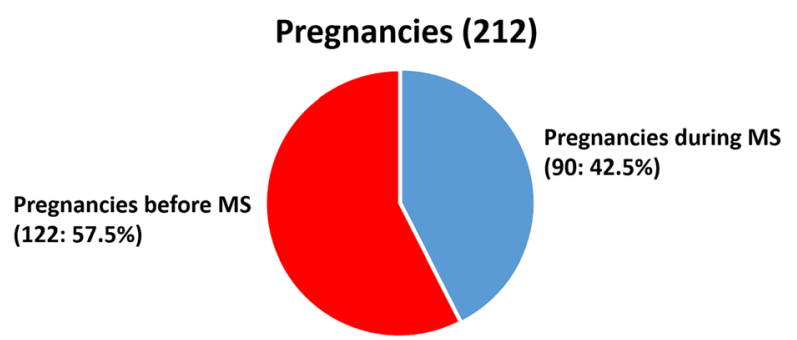

Fig. 1 Number of pregnancies categorised as pre-MS onset in red $(122 / 212 ; 57.5 \%)$ and during the MS course in blue (90/212; 42.5\%)

being higher in the patient group with pregnancy before MS onset versus the group with pregnancy after MS onset $(p<0.005)$ (Table 2$)$. Table 2 also details the follow-up period of each patient's cohort.

To evaluate the effect of pregnancy on shortterm MS outcomes, all pregnancies of the enrolled patients were examined $(n=212)$, with particular focus on pregnancies that occurred after MS onset (90 [42.4\%]) (Fig. 1). Of these, $32.2 \%$ of the patients received first-line DMTs during conception (12 with interferon-beta, 16 with glatiramer acetate, 1 with dimethyl fumarate), $8.8 \%$ were exposed to second-line DMTs (5 to natalizumab during MS and 3 to fingolimod before conception), while 59\% received no treatments. Of the 90 pregnancies examined during MS, a clinical relapse during pregnancy was observed in four patients: two after natalizumab discontinuation and one after fingolimod discontinuation. All relapses were treated with high-dose corticosteroids.

Following these pregnancies, in $45 \%$ of cases patients breastfed for at least 2 months; among these, $56 \%$ breastfed exclusively. As shown in Table 3, use of the $t$-test for paired samples revealed a higher ARR in the post-partum year compared with the pre-conception year $(0.54 \pm 0.84$ vs. $0.45 \pm 0.71 ; p=0.04)$ but no difference in EDSS score. Then, a multiple regression analysis was performed, showing a significant relationship in number of relapses at 12 months after childbirth (dependent variable) with clinical activity in the pre-conception year $(p=0.001)$ as well as with duration in months of breastfeeding $(p=0.022)$, much shorter in relation to the post-partum clinical activity.
Table 3 Effect of pregnancy on short-term clinical outcomes (number of relapses and EDSS pre and post pregnancy)

\begin{tabular}{llll}
\hline & $\begin{array}{l}\text { 1 year before } \\
\text { conception }\end{array}$ & $\begin{array}{l}\text { 1 year after } \\
\text { childbirth }\end{array}$ & $\boldsymbol{P}$ value \\
\hline $\begin{array}{l}\text { Number of } \\
\text { relapses } \\
(\text { mean } \pm \text { SD) }\end{array}$ & $0.45 \pm 0.71$ & $0.54 \pm 0.84$ & $\mathbf{0 . 0 4}$ \\
\hline
\end{tabular}

\begin{tabular}{llll}
\hline & At conception time & After childbirth & $\boldsymbol{P}$ value \\
\hline EDSS & $1.36 \pm 0.48$ & $1.46 \pm 0.78$ & ns
\end{tabular}

Bold value indicate the statistically significant data Paired samples $t$-tests were used to evaluate differences in number of relapses and EDSS score pre and post pregnancy

Moreover, higher clinical activity was observed in relation to second-line DMT exposure preconception or during pregnancy $(p=0.050)$. MRI activity in the post-partum year was related to the pre-conception MRI activity $(p=0.026)$, second-line DMT exposure pre-conception or during pregnancy $(p=0.041)$, and shorter breastfeeding duration $(p=0.013)$ (Table 4$)$. No relationships of clinical and MRI activity at 12 months after childbirth with exclusive breastfeeding were reported by regression analysis (data not shown).

To explore the possible influence of pregnancy and breastfeeding on long-term clinical and MRI outcomes, multiple regression models were also performed including EDSS score and MRI measurements obtained at the last neurological assessment (mean follow-up: $10.7 \pm 1.3$ years) as dependent variables. A lower EDSS score was associated with the presence of at least one pregnancy during MS $(p=0.021)$ after the correction for other clinical variables, disease duration $(p=0.001)$ and progressive course $(p=0.001)$ in particular (Table 5). Having had multiple pregnancies during MS was also related to a lower EDSS $(p=0.020)$. Conversely, a regression model showed no relationship between MRI measurements of WB, WM, and GM and pregnancy (Table 6a), while lower WM volumes were associated with a breastfeeding duration > 
Table 4 Short-term outcomes

\begin{tabular}{|c|c|c|c|c|}
\hline & \multirow[t]{2}{*}{ B } & \multicolumn{2}{|c|}{$\begin{array}{l}95 \% \text { CI for EXP } \\
\text { (B) }\end{array}$} & \multirow[t]{2}{*}{$\boldsymbol{P}$} \\
\hline & & Lower & Upper & \\
\hline \multicolumn{5}{|c|}{ Number of relapses, year after childbirth } \\
\hline $\begin{array}{l}\text { Relapses, year } \\
\text { before } \\
\text { conception }\end{array}$ & 0.665 & 0.411 & 0.918 & 0.001 \\
\hline $\begin{array}{l}\text { Duration of } \\
\text { breastfeeding } \\
\text { (months) }\end{array}$ & -0.053 & -0.098 & -0.008 & 0.022 \\
\hline $\begin{array}{l}\text { Second-line } \\
\text { DMD exposure } \\
\text { pre-conception }\end{array}$ & 0.385 & 0.192 & 0.214 & 0.050 \\
\hline \multicolumn{5}{|c|}{ MRI activity, year after childbirth } \\
\hline $\begin{array}{l}\text { MRI activity, } \\
\text { year before } \\
\text { conception }\end{array}$ & 0.319 & 0.041 & 0.598 & 0.026 \\
\hline $\begin{array}{l}\text { Duration of } \\
\text { breastfeeding }\end{array}$ & -0.052 & -0.093 & -0.012 & 0.013 \\
\hline $\begin{array}{l}\text { Second-line } \\
\text { DMDs } \\
\text { exposure } \\
\text { preconception }\end{array}$ & 0.321 & 0.043 & 0.560 & 0.031 \\
\hline
\end{tabular}

Bold values indicate the statistically significant data

Regression analysis. Relationships of clinical and MRI activity in the year after childbirth with the breastfeeding duration

Multiple linear regression analyses were used to examine the relationship between the number of relapses and MRI activity in the year after childbirth with the breastfeeding duration after controlling for clinical and MRI activity of the year before conception

6 months $(p=0.008)$ independent of other demographic and clinical variables (Table $6 \mathrm{~b}$ ).

Finally, a binary regression analysis was performed to explore factors influencing breastfeeding, showing that it was not conditioned by MS activity during the pre-conception year (ARR and MRI activity), while a relationship tending toward statistical significance was observed with exposure to second-line DMTs in
Table 5 Long-term outcomes

\section{EDSS score}

B $\quad 95 \%$ CI for EXP $P$

(B)

\begin{tabular}{lrrrr} 
& \multicolumn{3}{c}{ Lower } & \multicolumn{1}{c}{ Upper } \\
\cline { 3 - 4 } & & & \\
\hline Disease duration & 0.040 & 0.019 & 0.060 & $\mathbf{0 . 0 0 1}$ \\
Progressive MS & 3.181 & 2.548 & 3.814 & $\mathbf{0 . 0 0 1}$ \\
Pregnancy during & -0.424 & -0.783 & -0.064 & $\mathbf{0 . 0 2 1}$ \\
$\quad$ MS & & & & \\
\hline
\end{tabular}

Bold values indicate the statistically significant data

Multiple regression analysis. Relationships of EDSS score with MS clinical features and pregnancies during MS

Multiple linear regression analysis was used to examine the relationship of EDSS score, which was included in the model as dependent variable, with pregnancy during MS, while controlling for clinical MS variables (MS course and disease duration) and years of DMDs exposure

the pre-conception or pregnancy period $(p=0.07)$ (data not shown).

\section{DISCUSSION}

Pregnancy and the post-partum period represent important biological events that involve profound hormonal changes and have potential effects on MS activity and disease course [7, 23]. Consistently with other studies [7, 13], our research showed that the post-partum year is a phase with an increased risk of clinical relapses and MRI activity, which are conditioned by the clinical and neuroradiological activity of the pre-conception year and inversely related to breastfeeding duration. A recent meta-analysis of 24 studies that included nearly 3000 women concluded that breastfeeding is protective against post-partum relapses in MS. The association between breastfeeding and postpartum relapses appeared stronger in studies of exclusive breastfeeding for 2 months or more compared with studies including any breastfeeding in the breastfeeding group [19]. Classically, exclusive breastfeeding is defined as at least 2 months of breastfeeding without regular replacement of any meal by supplemental 
Table 6 Long-term outcomes

\begin{tabular}{|c|c|c|c|c|c|c|c|c|}
\hline \multicolumn{3}{|c|}{ (a) Whole brain } & \multicolumn{3}{|l|}{ White matter } & \multicolumn{3}{|l|}{ Grey matter } \\
\hline & B & $P$ value & & B & $P$ value & & B & $P$ value \\
\hline Age & -2.069 & 0.001 & Age & 0.225 & 0.472 & Age & -2.294 & 0.001 \\
\hline MS duration & -2.426 & 0.001 & MS duration & -0.850 & 0.033 & MS duration & -1.576 & 0.002 \\
\hline MS course & -26.672 & 0.186 & MS course & -1.710 & 0.875 & MS course & -24.966 & 0.077 \\
\hline Pregnancy & -7.161 & 0.370 & Pregnancy & -4.503 & 0.294 & Pregnancy & -2.659 & 0.632 \\
\hline \multicolumn{3}{|c|}{ (b) Whole brain } & \multicolumn{3}{|l|}{ White matter } & \multicolumn{3}{|l|}{ Grey matter } \\
\hline & B & $P$ value & & B & $P$ value & & B & $P$ value \\
\hline Age & -2.126 & 0.001 & Age & 0.165 & 0.588 & Age & -2.291 & 0.001 \\
\hline MS duration & -2.263 & 0.002 & MS duration & -0.694 & 0.073 & MS duration & -1.569 & 0.002 \\
\hline MS course & -28.90 & 0.151 & MS course & -3.651 & 0.733 & MS course & -25.25 & 0.074 \\
\hline Breastfeeding & -23.28 & 0.067 & Breastfeeding & -17.99 & 0.008 & Breastfeeding & -5.292 & 0.551 \\
\hline
\end{tabular}

Multiple regression analyses. Relationship of MRI measurements with demographics, clinical features, previous pregnancy, and duration of breastfeeding

Multiple linear regression analysis was used to examine the relationship between brain volumes (whole brain [WB], white matter [WM], grey matter [GM]), which were included in the model as dependent variables, previous pregnancy and breastfeeding duration during MS, while controlling for demographic (sex, age) and clinical variables (MS course and disease duration)

feeding [19]. With exclusive breastfeeding, an elevation in prolactin was reported and larger drops in follicle-stimulating hormone, luteinizing hormone, progesterone, and estradiol. All these hormonal changes work together to produce more prolonged amenorrhea and anovulation, with potentially beneficial immunological effects [19]. However, exclusive breastfeeding was observed in $56 \%$ of our breastfeeding women in the first 2 months, with no effects on clinical and MRI outcomes at 12 months. However, given the retrospective nature of the study, the data on exclusive breastfeeding may be fallacious. Less post-partum clinical activity was observed in our sample in women who breastfed longer; this may not be attributable to a protective role of lactation on MS activity but rather to a milder disease course in these women. In fact, women with higher MS activity likely had to renounce or prematurely quit breastfeeding to start DMT. Thus, breastfeeding duration seems more likely to be conditioned by MS, resulting it shorter durations among subjects with clinical or neuroradiological reactivation. In addition, even though we did not identify a relationship between the choice to forego breastfeeding and pre-pregnancy MS activity, we observed a relationship tending toward statistical significance with exposure to second-line DMTs pre-conception or during pregnancy. Therefore, it is likely that these women had to give up breastfeeding to resume second-line DMTs immediately after delivery. Accordingly, regarding short-term outcomes, a relationship between post-partum MS reactivation and pre-conception second-line DMT exposure was found, indicating that these women are the most prone to the risk of disease recurrence. This may be attributable to the most active form of MS as well as to disease reactivation linked to the discontinuation of more effective therapies $[11,24]$. However, given that pre-conception second-line DMT exposure was observed only in $8.8 \%$ of pregnancies, the small sample size limits the power of this result. Recently, a study 
performed in MS patients that discontinued natalizumab for pregnancy-related reasons has shown that recurrence of disease activity, clinically and/or radiologically, was observed in 95\% of discontinuations, although all patients showed no or limited disease activity in the year preceding natalizumab discontinuation [25]. However, of the cases in whom conception occurred under natalizumab treatment, fewer relapses and less radiological activity were observed [25]. Thus, continuation of treatment until conception may be a preferred strategy to prevent relapses and MRI activity in women on natalizumab treatment who want to get pregnant. In line with this new evidence, in the last few years the approach to pregnancy has changed. The most recent recommendations of the European Academy of Neurology and European Committee of Treatment of Research in MS have better addressed DMT use during conception and pregnancy [26], especially in light of new data about drug safety. While the discontinuation of fingolimod until conception is necessary for safety reasons, treatment with natalizumab during the first two trimesters of pregnancy should be considered [27], with immediate post-partum resumption at the expense of breastfeeding, despite preliminary evidence of its safe use during this phase [28]. Other recommendations about more effective and recently launched DMTs are constantly evolving, making MS treatment during this biological reproductive phase increasingly centred on the patient and her MS characteristics [29]. However, the long-term effects of pregnancy and puerperium on MS are extremely difficult to measure. A recent intriguing study by Portaccio et al. evaluated the influence of the pre-pregnancy period on long-term disability in MS and observed that the risk of long-term disability is higher among MS women with relapses in the pre-conception year [30], reinforcing the importance of pregnancy planning and treatment optimisation during this reproductive phase. Thus, new data on long-term outcomes are urgently needed to better understand how to best orient therapeutic choices in the era of new DMTs. In line with some studies that reported lower disability scores in women with previous pregnancies compared with nulliparous women $[23,30]$, our study focused on long-term outcomes and showed that having had at least one pregnancy was associated with lower EDSS score independent of other factors. More so, this effect is observed in the case of multiple pregnancies. However, although pregnancy has classically been considered a selftreatment due to the state of immunotolerance that it creates, its protective role against longterm disability progression is difficult to establish. Conversely, in other words, instead of the protective effect of pregnancy, women with more aggressive MS forms may be less willing to have or be discouraged from having children. A previous French and Italian cohort study provided an original description of the evolution of MS activity after two successive pregnancies in women with MS, showing similar (and even lower) disease activity in the second versus first pregnancy [31]. This could be due to the fact that, if MS women were generally advised to consider pregnancy in a quiet period of the disease, women who experienced a relapse during a first pregnancy or after delivery may fear a similar outcome and be discouraged from considering a second pregnancy. In line with this, it is likely that women with MS with multiple pregnancies are also those with milder MS [31]; assuming that higher disease activity is related to worse long-term disability, they could have better long-term clinical outcomes.

Furthermore, it should be highlighted that pregnancy is a well-circumscribed biological event in a long history of disease and that our patients had heterogeneous MS characteristics and DMT exposures before and after pregnancy, which complicates our evaluation of outcomes. Another central point is whether pregnancy in MS can have a protective effect against brain damage and, in particular, the evolution of brain atrophy, increasingly considered the final expression of MS brain injury. Khalid et al. previously performed a quantitative MRI analysis of cerebral lesions and atrophy in postpartum patients with MS and reported no WB or cortical atrophy despite increases in destructive lesions in the immediate post-partum period; thus, they emphasised the need for longer follow-up durations to verify these findings [32]. Set in this context, our study evaluated long- 
term brain atrophy and found no correlation among WB, WM, and GM volumes and a history of pregnancies either before or after MS onset. Interestingly, however, our study showed that breastfeeding for $>6$ months was associated with lower WM volume. This finding draws attention to the importance of the post-partum period and the choice of breastfeeding, which until very recently corresponded to the choice of postponing DMT use, exposing the woman to MS reactivation with immediate effects such as those in the post-partum period or the long term. These effects can be reversible, such as clinical relapses and MRI inflammatory activity, or permanent, such as WM atrophy, likely attributable to the inflammatory processes that occur in the post-partum period.

However, today's therapeutic landscape has changed; in particular, interferon-beta was recently approved for use during lactation by the European Medicines Administration and the US Food and Drug Administration based on safety data [33]; new evidence of the safety of other MS drugs is also on the near horizon.

In addition to the previously discussed limitations mainly related to sample size and heterogeneity, other limitations require addressing. As discussed, the patients had different follow-up times, MS characteristics, and DMT exposures (previous and concomitant). These aspects may have affected the brain volume measurements in particular. Moreover, it is known that cerebral atrophy represents a complex outcome that is related with demographics, clinical aspects, and MS-unrelated factors (i.e., comorbidities and lifestyle) [34], which were not specifically evaluated in this study. Moreover, the study was performed before the launch of some DMTs (e.g., B cell depletors, newer S1P receptor modulators), and this makes some results less generalisable.

Finally, since this study focused on MS-related outcomes, foetal risks and outcomes of the examined pregnancies were not evaluated.

\section{CONCLUSIONS}

Our data confirm the previous findings about MS reactivation risk in the post-partum period, in particular in relation to the pre-conception clinical and neuroradiological activity as well as exposure to second-line DMTs and the possible effect of DMT discontinuation on MS activity. These findings reinforce the importance of adequate counselling and pregnancy planning to optimise therapeutic choices [35] and the window of therapeutic opportunity based on new evidence of DMT safety [34]. In fact, the therapeutic choices made during the reproductive biological phases of pregnancy and puerperium could affect long-term MS outcomes even more than pregnancy and prolonged breastfeeding affect MS-related disability and brain atrophy.

In this era of new DMTs, new studies are needed to better understand the short- and long-term effects of pregnancy and puerperium in women with MS as well as the best pharmacological management of these biological phases so central in women's lives.

\section{ACKNOWLEDGEMENTS}

Funding. The study was supported by Fondazione di Sardegna. The journal's Rapid Service Fee was funded by the authors.

Authorship. All named authors meet the International Committee of Medical Journal Editors (ICMJE) criteria for authorship for this article, take responsibility for the integrity of the work as a whole, and have given their approval for this version to be published.

Authors' Contributions. Lorefice Lorena: study design, statistical analysis, data interpretation, drafting and revision of the manuscript. Fronza Marzia: study design, acquisition of data, drafting and revision of the manuscript. Fenu Giuseppe, Frau Jessica, Coghe Giancarlo, Murgia Federica, D'Alterio Maurizio Nicola, Angioni Stefano, Barracciu Maria Antonietta: data acquisition and interpretation, revision of the manuscript. Cocco Eleonora: study design, critical revision of the manuscript and supervision. 
Disclosures. Lorena Lorefice, Jessica Frau, Giuseppe Fenu, Giancarlo Coghe, Eleonora Cocco received honoraria for consultancy or speaking from Biogen, Novartis, Sanofi, Genzyme, Serono and Teva and Almirall. Marzia Fronza, Federica Murgia, Maria Antonietta Barracciu, Maurizio Nicola, D'Alterio and Stefano Angioni have nothing to disclose.

Compliance with Ethics Guidelines. The local ethics committee of ATS Sardegna, Sassari, approved the study (protocol 206/2020/EC-18 February 2020). Informed consent was obtained from all participants after the local ethics committee approval.

Data Availability. The raw data supporting the conclusions of this article will be made available by the authors, without undue reservation.

Open Access. This article is licensed under a Creative Commons Attribution-NonCommercial 4.0 International License, which permits any non-commercial use, sharing, adaptation, distribution and reproduction in any medium or format, as long as you give appropriate credit to the original author(s) and the source, provide a link to the Creative Commons licence, and indicate if changes were made. The images or other third party material in this article are included in the article's Creative Commons licence, unless indicated otherwise in a credit line to the material. If material is not included in the article's Creative Commons licence and your intended use is not permitted by statutory regulation or exceeds the permitted use, you will need to obtain permission directly from the copyright holder. To view a copy of this licence, visit http://creativecommons.org/licenses/by$\mathrm{nc} / 4.0 /$.

\section{REFERENCES}

1. Nair RR, Verma P, Singh K. Immune- endocrine crosstalk during pregnancy. Gen Comp Endocrinol. 2017;242:18-23.
2. Airas L, Saraste M, Rinta S, et al. Immunoregulatory factors in multiple sclerosis patients during and after pregnancy: relevance of natural killer cells. Clin Exp Immunol. 2008;151(2):235-43.

3. De Las HV, De Andres C, Tellez N, et al. Pregnancy in multiple sclerosis patients treated with immunomodulators prior to or during part of the pregnancy: a descriptive study in the Spanish population. Mult Scler. 2007;13:981-4.

4. Fernández Liguori N, Klajn D, et al. Epidemiological characteristics of pregnancy, delivery, and birth outcome in women with multiple sclerosis in Argentina (EMEMAR study). Mult Scler. 2009;15(5): 555-62.

5. Finkelsztejn A, Fragoso YD, Ferreira ML, et al. The Brazilian database on pregnancy in multiple sclerosis. Clin Neurol Neurosurg. 2011;113(4):277-80.

6. Confavreux C, Hutchinson M, Hours MM, et al. Rate of pregnancy-related relapse in multiple sclerosis. Pregnancy in Multiple Sclerosis Group. N Engl J Med. 1998;339(5):285-91.

7. Dobson R, Jokubaitis VG, Giovannoni G. Change in pregnancy-associated multiple sclerosis relapse rates over time: a meta-analysis. Mult Scler Relat Disord. 2020;44:102241.

8. Haghikia A, Langer-Gould A, Rellensmann G, et al. Natalizumab use during the third trimester of pregnancy. JAMA Neurol. 2014;71(7):891-5.

9. Meinl I, Havla J, Hohlfeld R, et al. Recurrence of disease activity during pregnancy after cessation of fingolimod in multiple sclerosis. Mult Scler. 2018;24(7):991-4.

10. Novi G, Ghezzi A, Pizzorno $M$, et al. Dramatic rebounds of MS during pregnancy following fingolimod withdrawal. Neurol Neuroimmunol Neuroinflamm. 2017;4(5):e377.

11. Sepúlveda M, Montejo C, Llufriu S, et al. Rebound of multiple sclerosis activity after fingolimod withdrawal due to planning pregnancy: analysis of predisposing factors. Mult Scler Relat Disord. 2020;38: 101483.

12. Kurtzke JF. Rating neurologic impairment in multiple sclerosis: an expanded disability status scale (EDSS). Neurology. 1983;33(11):1444-52.

13. Hughes SE, Spelman T, Gray OM, et al. Predictors and dynamics of postpartum relapses in women with multiple sclerosis. Mult Scler. 2014;20(6): 739-46.

14. Langer-Gould A, Smith JB, Albers KB, et al. Pregnancy-related relapses and breastfeeding in a 
contemporary multiple sclerosis cohort. Neurology. 2020;94(18):e1939-49.

15. Hellwig K, RockhoffM HS, et al. Exclusive breastfeeding and the effect on postpartum multiple sclerosis relapses. JAMA Neurol. 2015;72:1132-8.

16. Langer-Gould A, Huang SM, Gupta R, et al. Exclusive breastfeeding and the risk of postpartum relapses in women with multiple sclerosis. Arch Neurol. 2009;66:958-63.

17. Portaccio E, Ghezzi A, Hakiki B, et al. Breastfeeding is not related to postpartum relapses in multiple sclerosis. Neurology. 2011;77:145-50.

18. Jesus-Ribeiro J, Correia I, Martins AI, et al. Pregnancy in multiple sclerosis: a Portuguese cohort study. Mult Scler Relat Disord. 2017;17:63-8.

19. Krysko KM, Rutatangwa A, Graves J, et al. Association between breastfeeding and postpartum multiple sclerosis relapses: a systematic review and metaanalysis. JAMA Neurol. 2020;77(3):327-38.

20. Portaccio E, Amato MP. Breastfeeding and postpartum relapses in multiple sclerosis patients. Mult Scler. 2019;25(9):1211-6.

21. Thompson AJ, Banwell BL, Barkhof F, et al. Diagnosis of multiple sclerosis: 2017 revisions of the McDonald criteria. Lancet Neurol. 2018;17(2): 162-73.

22. Smith SM, Zhang Y, Jenkinson M, et al. Accurate, robust, and automated longitudinal and cross-sectional brain change analysis. Neuroimage. 2002;17(1):479-89.

23. D'Hooghe MB, Nagels G, Uitdehaag BM. Long-term effects of childbirth in MS. J Neurol Neurosurg Psychiatry. 2010;81:38-41.

24. De Giglio L, Gasperini C, Tortorella C, et al. Natalizumab discontinuation and disease restart in pregnancy: a case series. Acta Neurol Scand. 2015;131(5):336-40.

25. Kleerekooper I, van Kempen ZLE, Leurs CE, et al. Disease activity following pregnancy-related discontinuation of natalizumab in MS. Neurol Neuroimmunol Neuroinflamm. 2017;5(1):e424.

26. Montalban X, Gold R, Thompson AJ, et al. ECTRIMS/EAN guideline on the pharmacological treatment of people with multiple sclerosis. Mult Scler. 2018;24(2):96-120.

27. Portaccio E, Moiola L, Martinelli V, et al. Pregnancy decision-making in women with multiple sclerosis treated with natalizumab: II: maternal risks. Neurology. 2018;90(10):e832-9.

28. Landi D, Marfia GA. Exposure to natalizumab during pregnancy and lactation is safe-yes. Mult Scler. 2020;26(8):887-9.

29. Portaccio E, Tudisco L, Pastò L, et al. Pregnancy in multiple sclerosis women with relapses in the year before conception increases the risk of long-term disability worsening. Mult Scler. 2021. https://doi. org/10.1177/13524585211023365.

30. Jokubaitis VG, Spelman T, Kalincik T, et al. Predictors of long-term disability accrual in relapse-onset multiple sclerosis. Ann Neurol. 2016;80(1):89-100.

31. Benoit A, Durand-Dubief F, Amato MP, et al. History of multiple sclerosis in 2 successive pregnancies: a French and Italian cohort. Neurology. 2016;87(13):1360-7.

32. Khalid F, Healy BC, Dupuy SL, et al. Quantitative MRI analysis of cerebral lesions and atrophy in post-partum patients with multiple sclerosis. J Neurol Sci. 2018;392:94-9.

33. Ciplea AI, Langer-Gould A, Stahl A, et al. Safety of potential breast milk exposure to IFN- $\beta$ or glatiramer acetate: one-year infant outcomes. Neurol Neuroimmunol Neuroinflamm. 2020;7(4):e757.

34. Lorefice L, Frau J, Coghe G, et al. Assessing the burden of vascular risk factors on brain atrophy in multiple sclerosis: a case-control MRI study. Mult Scler Relat Disord. 2019;27:74-8.

35. Krysko KM, Bove R, Dobson R, et al. Treatment of women with multiple sclerosis planning pregnancy. Curr Treat Options Neurol. 2021;23(4):11. 